\title{
Portal Biliopathy Diagnosed Using Color Doppler and Contrast-enhanced Ultrasound
}

\author{
Hiroaki Nunoi ${ }^{1}$, Masashi Hirooka ${ }^{1}$, Hironori Ochi ${ }^{1}$, Yohei Koizumi ${ }^{1}$, Yoshio Tokumoto ${ }^{1}$, \\ Masanori Abe ${ }^{1}$, Fujimasa Tada ${ }^{1}$, Yoshio Ikeda ${ }^{1}$, Bunzo Matsuura ${ }^{1}$, Hiroaki Tanaka ${ }^{2}$, \\ Takaharu Tsuda $^{2}$, Teruhito Mochizuki ${ }^{2}$, Yoichi Hiasa ${ }^{1}$ and Morikazu Onji ${ }^{1}$
}

\begin{abstract}
Portal biliopathy is a morphological abnormality of the biliary ductal and gallbladder wall associated with portal hypertension. A patient with essential thrombocythemia was initially diagnosed with extrahepatic portal vein obstruction (EHPVO). The contrast-enhanced computed tomography (CT) findings were similar to those of cholangiocarcinoma or sclerosing cholangitis. However, color Doppler and contrast-enhanced ultrasound (US) were more specific. The paracholedocheal veins around the bile ducts appeared as beads soon after the injection of contrast medium, followed by linear enhancement of the epicholedochal veins and the gradual enhancement of the whole bile ducts. These findings led to a diagnosis of portal biliopathy, which prevented the patient from having to endure hazardous procedures such as bile duct biopsies. Color Doppler and contrast-enhanced US findings are useful for diagnosing or ruling out portal biliopathy in patients who present with EHPVO.
\end{abstract}

Key words: extrahepatic venous obstruction, essential thrombocythemia, pseudocholangiocarcinoma

(Intern Med 52: 1055-1059, 2013)

(DOI: 10.2169/internalmedicine.52.8848)

\section{Introduction}

Portal biliopathy can arise in patients with extrahepatic portal vein obstruction (EHPVO), which can be caused by clotting disorders, abdominal postoperative complications, malignant tumors, dehydration and neonatal umbilical vein catheterization (1). Pseudosclerosing cholangitis and pseudocholangiocarcinoma are biliary ductal and gallbladder wall abnormalities associated with portal hypertension. If portal biliopathy is misdiagnosed as pseudosclerosing cholangitis or pseudocholangiocarcinoma, performing a bile duct biopsy and pancreatic head resection is necessary but hazardous. Such procedures can be avoided using imaging modalities to prevent complications (2).

We herein describe the case of a patient with portal biliopathy diagnosed on color Doppler and contrastenhanced ultrasound (US), which eliminated the need for a biopsy or surgery.

\section{Case Report}

Upper gastrointestinal endoscopy revealed bleeding from esophageal varices in a 55-year-old woman with melena who was referred to our hospital for treatment. The patient was in a hypercoagulable state resulting from essential thrombocythemia (Table); however, her medical history and that of her family were otherwise unremarkable and did not include abdominal surgery.

The patient was alert at the time of admission with a normal liver function. Contrast-enhanced computed tomography (CT) of the abdomen revealed a slightly enhanced solid mass around the common bile duct and ductal narrowing in the portal phase (Fig. 1). The intrahepatic bile duct and pancreatic duct were not dilated, although the gallbladder wall had thickened. We considered the major portal and splenic

${ }^{1}$ Department of Gastroenterology and Metabology, Ehime University Graduate School of Medicine, Japan and ${ }^{2}$ Department of Diagnostic and Therapeutic Radiology, Ehime University Graduate School of Medicine, Japan

Received for publication August 23, 2012; Accepted for publication January 16, 2013

Correspondence to Dr. Yoichi Hiasa, hiasa@m.ehime-u.ac.jp 
Table. Laboratory Data on Admission

\begin{tabular}{|c|c|c|c|c|c|c|c|c|}
\hline White blood cell & 6,000 & $/ \mu \mathrm{L}$ & Total protein & 7.2 & $\mathrm{~g} / \mathrm{dL}$ & Hepatitis B surface antigen & & $(-)$ \\
\hline Red blood cell & $434 \times 10^{4}$ & $/ \mu \mathrm{L}$ & Albumin & 3.9 & $\mathrm{~g} / \mathrm{dL}$ & anti-hepatitis $\mathrm{C}$ virus antibody & & $(-)$ \\
\hline Hemoglobin & 12.2 & $\mathrm{~g} / \mathrm{dL}$ & Total bilirubin & 0.9 & $\mathrm{mg} / \mathrm{dL}$ & & & \\
\hline Hematocrit & 37.5 & $\%$ & Direct bilirubin & 0.2 & $\mathrm{mg} / \mathrm{dL}$ & Occult blood & & $(+)$ \\
\hline \multirow[t]{2}{*}{ Platelet } & $59.8 \times 10^{4}$ & $/ \mu \mathrm{L}$ & Aspartate aminotransferase $*$ & 26 & $\mathrm{IU} / \mathrm{L}$ & & & \\
\hline & & & Alanine aminotransferase $*$ & 23 & $\mathrm{IU} / \mathrm{L}$ & & & \\
\hline \multirow[t]{2}{*}{ Erythrocyte sedimentation rate } & 7.3 & $\mathrm{~mm} / \mathrm{h}$ & Lactate dehydrogenase & 269 & $\mathrm{IU} / \mathrm{L}$ & PIVKA-II & 17 & $\mathrm{mAU} / \mathrm{mL}$ \\
\hline & & & Alkaline phosphatase * & 354 & $\mathrm{IU} / \mathrm{L}$ & Alfa-Fetoprotein & 2.5 & $\mathrm{ng} / \mathrm{mL}$ \\
\hline Activated partial thromboplastin time & 25.7 & $\sec$ & Gammaglutamyl transpeptidase * & 57 & $\mathrm{IU} / \mathrm{L}$ & CEA & 0.8 & $\mathrm{ng} / \mathrm{mL}$ \\
\hline Prothrombin time & 89.0 & $\%$ & Leucine aminopeptidase $*$ & 56 & $\mathrm{IU} / \mathrm{L}$ & CA19-9 & 4.8 & $\mathrm{U} / \mathrm{mL}$ \\
\hline INR & 1.12 & & Cholinesterase & 291 & $\mathrm{IU} / \mathrm{L}$ & & & \\
\hline Anti-thrombin III & 102.2 & $\%$ & Zinc sulfate turbidity test & 9 & $\mathrm{U}$ & & & \\
\hline Fibrinogen & 295 & $\mathrm{mg} / \mathrm{dL}$ & Thymol turbidity test & 10 & $\mathrm{U}$ & Immunoglobulin $\mathrm{G}$ & 1,220 & $\mathrm{mg} / \mathrm{dL}$ \\
\hline \multirow[t]{2}{*}{ Fibrinogen degradation product } & 4.1 & $\mu \mathrm{g} / \mathrm{mL}$ & Total cholesterol & 203 & $\mathrm{mg} / \mathrm{dL}$ & Immunoglobulin A & 294 & $\mathrm{mg} / \mathrm{dL}$ \\
\hline & & & Triglyceride & 158 & $\mathrm{mg} / \mathrm{dL}$ & Immunoglobulin $\mathrm{M}$ & 107 & $\mathrm{mg} / \mathrm{dL}$ \\
\hline
\end{tabular}

*Normal range: aspartate aminotransferase, 9-37 IU/L; alanine aminotransferase, 3-49 IU/L; platelet, 13.1-36.9 $\times 10^{4} / \mu \mathrm{L}$


Figure 1. Contrast-enhanced computed tomography of the abdomen. The images show the superior mesenteric, portal and splenic veins at the arterial (A) and portal (B) phases. The mass in the common bile duct is slightly enhanced in the portal, but not the arterial, phase (white arrow). The intrahepatic bile duct is not dilated. The gallbladder neck is enhanced (black arrow), whereas the absence of enhancement in the major portal trunk (white arrowhead) indicates portal obstruction by a thrombus.

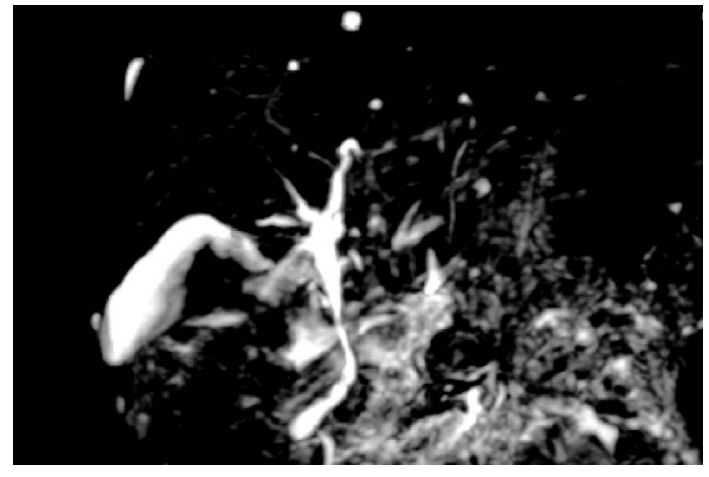

Figure 2. T2-weighted magnetic resonance cholangiography images. The common bile duct is narrow.

veins to be obstructed, as they were not enhanced. Magnetic resonance cholangiography also showed narrowing of the common bile duct lumen (Fig. 2) and abdominal US in B mode revealed obvious thickening of the common bile duct wall. The common bile duct was still patent, despite remark- able luminal narrowing. The intrahepatic bile duct was not dilated. Liver echogenicity was normal on US images (Fig. 3). Color Doppler ultrasound identified the vascular nature of the mass and a network of serpentine vessels with a hepatopetal flow comprising the portoportal collaterals in the hepatoduodenal ligament and porta hepatis. Varices of the gallbladder appeared as tortuous dilated vessels around the wall. Based on these findings, we considered a diagnosis of portal cavernoma around the common bile duct and ruled out cholangiocarcinoma or sclerosing cholangitis. The common bile duct wall was remarkably enhanced on CT images acquired during arterial portography, and gallbladder varices were obvious (Fig. 4). We therefore made a diagnosis of portal biliopathy and gallbladder varices caused by EHPVO with essential thrombocythemia. The esophageal varices were treated with sclerotherapy. The histological findings of a liver biopsy were essentially normal, and hepatic fibrosis was absent (Fig. 5). We also performed contrast-enhanced US. The paracholedocheal veins around the bile ducts appeared as beads soon after the injection of contrast medium, 

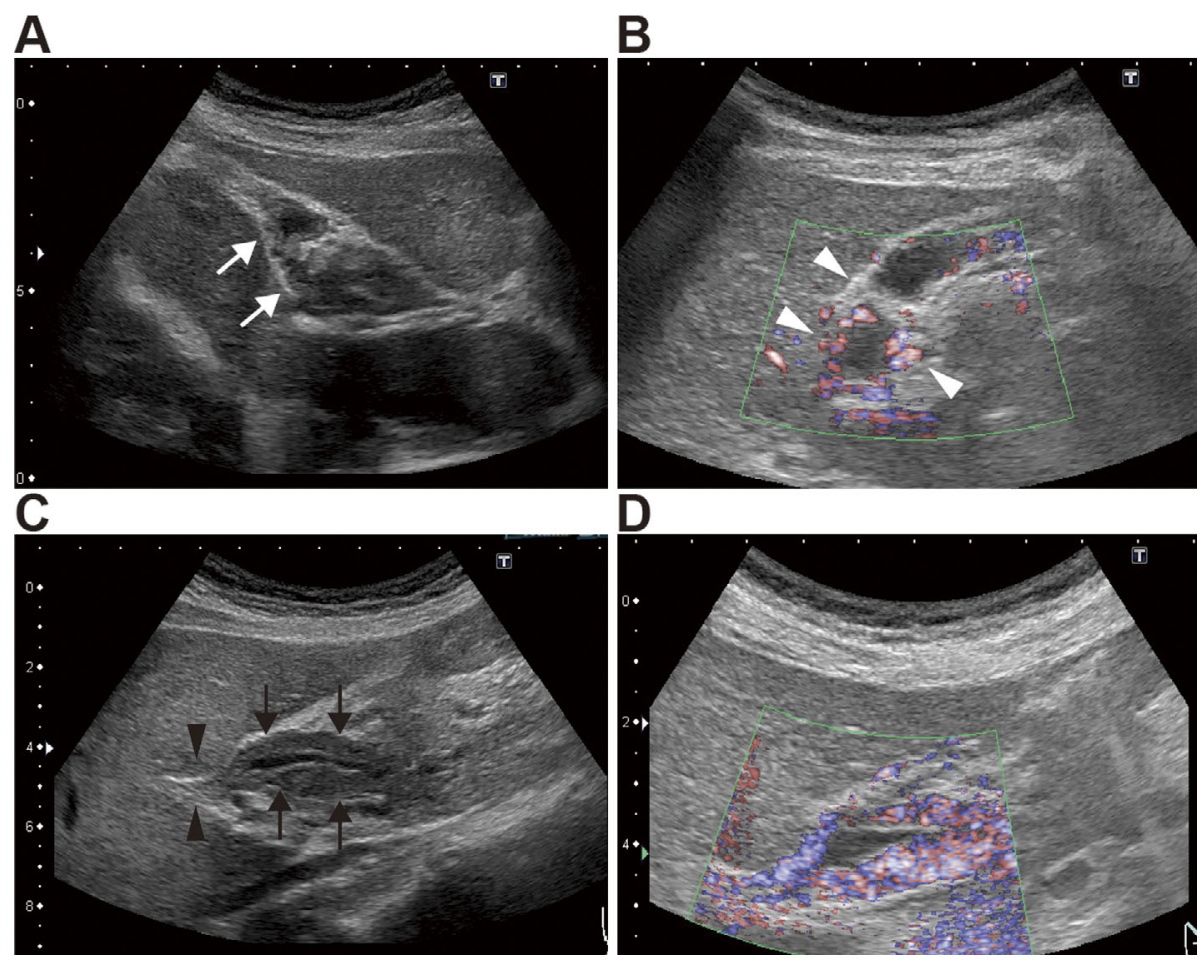

Figure 3. Ultrasound findings. Doppler US reveals tortuous dilated vessels around a slightly thickened gallbladder wall (white arrow, A), indicating varices (white arrowhead, B). The wall of the common bile duct is also thickened (black arrow, $\mathrm{C}$ ). The intrahepatic bile duct is not dilated (black arrowhead). Doppler US clearly shows a vascular lesion (D).
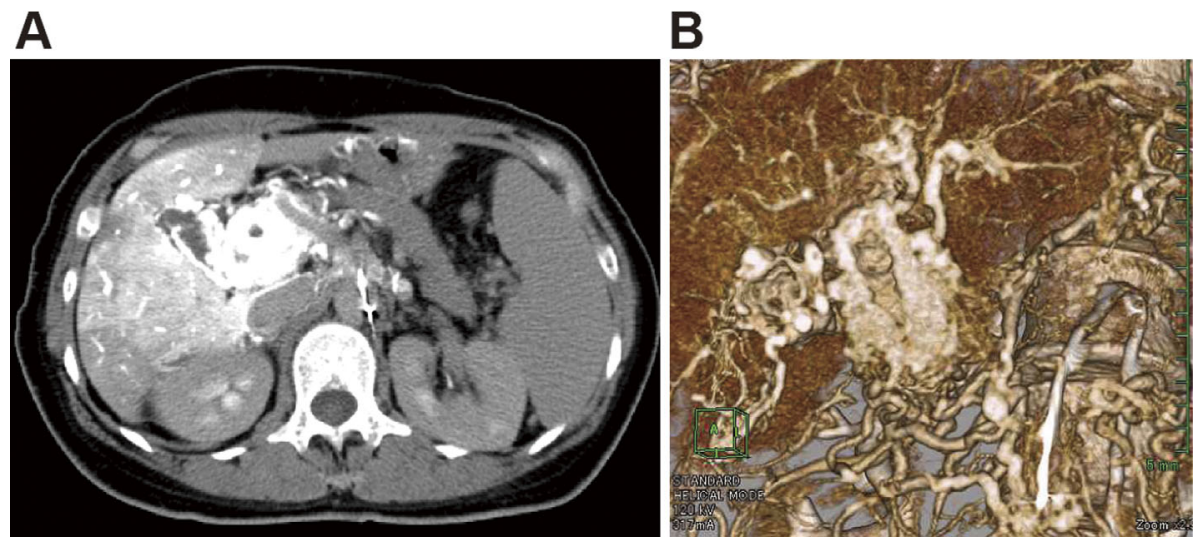

Figure 4. Computed tomography (A) and three-dimensional (B) imaging during arterial portography. The wall of the common bile duct is remarkably thickened (A) and gallbladder varices are obvious on 3D images (B).

followed by linear enhancement of the epicholedochal veins and gradual enhancement of the whole bile ducts. Both the epicholedochal and paracholedochal venous plexuses were dilated (Fig. 6); these findings confirmed the diagnosis of portal biliopathy. We treated the essential thrombocythemia with hydrocarbamide and followed up the portal biliopathy using imaging modalities.

\section{Discussion}

Portal biliopathy includes abnormalities of the entire biliary tract, including the intrahepatic and extrahepatic bile and cystic ducts, as well as the gallbladder, in patients with portal hypertension. The drainage veins of the common bile duct form an epicholedochal and paracholedochal venous plexus (2-4). Hypertrophy of both collateral plexuses produces a scalloped or smooth indentation in the ductal lumen of the bile duct, which can progress to more dramatic narrowing, stenosis and kinking. The development of gallbladder varices as a collateral pathway via the cystic vein is also a characteristic feature of portal biliopathy (3). Although the prevalence of this condition, and of EHPVO in particular, is still not understood in detail, elevated hepatobiliary enzymes and cholangiographic abnormalities are considered to be 
A

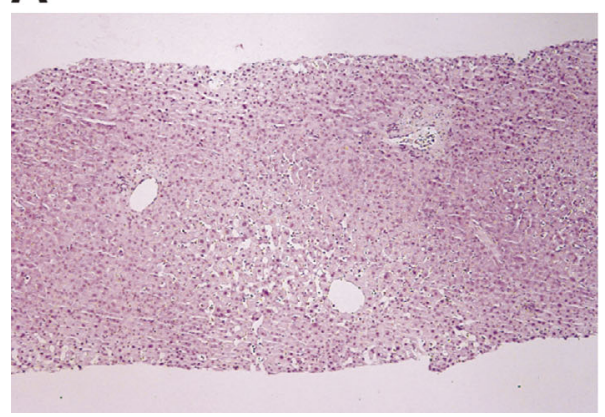

B

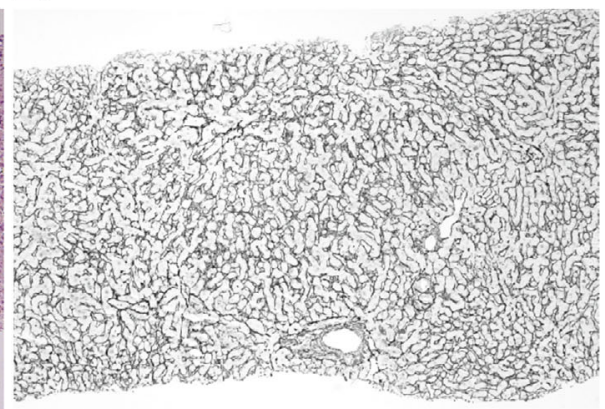

Figure 5. Histological findings of the liver specimen. The findings of Hematoxylin and Eosin staining $(A)$ and silver $(B)$ staining are normal (magnification $\times 40)$.

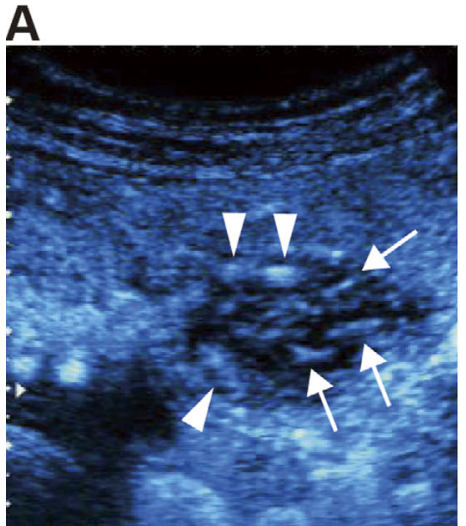

$40 \mathrm{sec}$
B

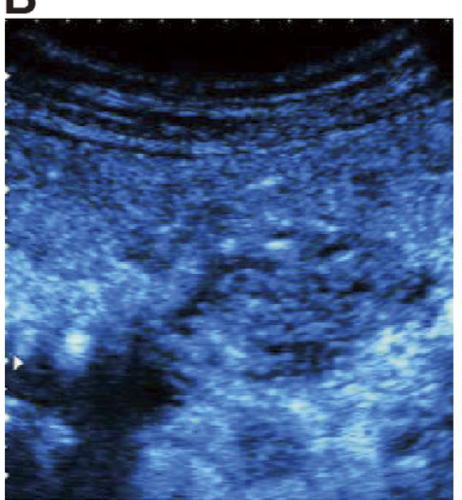

$42 \mathrm{sec}$

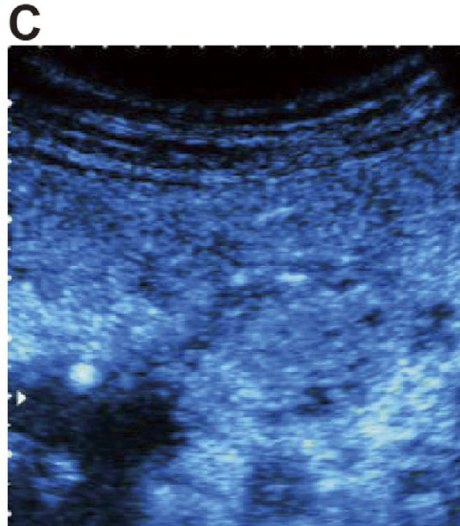

$45 \mathrm{sec}$

After injection of contrast medium

Figure 6. Contrast-enhanced ultrasound imaging findings. Dilation of the epicholedochal and/or paracholedochal venous plexuses indicates portal biliopathy. The paracholedocheal veins around the bile ducts initially became enhanced to resemble beads (arrowheads), then the epicholedochal veins immediately became linearly enhanced (white arrows, A). Thereafter, the whole bile ducts gradually became enhanced $(B, C)$. Contrast-enhanced US clearly differentiates dilation of the paracholedochal (arrowheads) and epicholedochal (white arrows) venous plexuses.

commonly associated in patients with portal hypertension. Cholangiographic abnormalities, liver dysfunction and cholangitis are found in $81 \%-93 \%, 40 \%-67 \%$ and $5 \%-18 \%$ of patients with EHPVO, respectively (5-7). The cholangiographic findings sometimes mimic bile duct cancer, with cavernomas appearing as solid tumors that are considered to be a sign of pseudocholangiocarcinoma (8). Although vascular diseases can be diagnosed on dynamic CT, the contrast patterns of the collateral and normal vessels differed in this patient. Vascular abnormalities could not be differentiated in our patient due to a weak enhancement in the portal phase (Fig. 1). The reasons for the poor enhancement remain obscure; however, we speculated that the amount of contrast medium would be diminished and would reach the epicholedochal plexus, which comprises a mesh of venous vessels of $<1 \mathrm{~mm}$ in diameter, at different times. Moreover, such inconsistencies might also be responsible for the irregularity and tortuosity of the paracholedochal venous plexus. If the findings indicate bile duct cancer, then the patients might have to undergo risky maneuvers, such as biliary biopsies.
Patients should initially be examined using various diagnostic imaging modalities such as color Doppler and contrastenhanced US to avoid the need for hazardous and unnecessary procedures (2). Color Doppler (3) and contrastenhanced US can also avoid misdiagnosing porto-portal collateral veins as solid masses on gray-scale images by defining the vascular nature of the abnormalities. The drainage veins of the common bile duct form an epicholedochal plexus that appears as a mesh of venous vessels with a diameter of $<1 \mathrm{~mm}$ on the surface of the common bile and hepatic ducts, and a paracholedochal venous plexus runs parallel to the bile duct. Contrast-enhanced US can distinguish between these two collateral plexuses (Fig. 6). Hypertrophic changes in the venous plexus have not been reported as far as we can determine. The resolution of color Doppler US is too low to distinguish these venous plexuses, whereas contrast-enhanced US can clearly differentiate them. Gallbladder varices appear as tortuous dilated vessels in or around the gallbladder wall and can be easily diagnosed using color Doppler or contrast-enhanced US. 
Our patient developed a biliary abnormality without exhibiting any other symptoms, such as pruritus, jaundice, fever, abdominal pain or fever. Only the esophageal varices were treated with sclerosing therapy. The symptoms of portal biliopathy are associated with a more advanced age and a longer disease duration (9). Providing follow-up is necessary because more symptoms might emerge over time.

In conclusion, we herein described a case of portal biliopathy that was visualized on contrast-enhanced and color Doppler US. When patients present with portal hypertension, both contrast-enhanced and color Doppler US are thus considered to be useful modalities for making a differential diagnosis of portal biliopathy.

The authors state that they have no Conflict of Interest (COI).

\section{References}

1. Valla D, Casadevall N, Huisse MG, et al. Etiology of portal vein thrombosis in adults. A prospective evaluation of primary myeloproliferative disorders. Gastroenterology 94: 1063-1069,
1988.

2. Walser EM, Runyan BR, Heckman MG, et al. Extrahepatic portal biliopathy: Proposed etiology on the basis of anatomic and clinical features. Radiology 258: 146-153, 2011.

3. Besa C, Cruz PJ, Huete A, Cruz F. Portal biliopathy: multitechnique imaging approach. Abdom Imaging 37: 83-90, 2012.

4. Shin SM, Kim S, Lee JW, et al. Biliary abnormalities associated with portal biliopathy: Evaluation on MR cholangiography. AJR Am J Roentgenol 188: 341-347, 2007.

5. Khuroo MS, Yattoo GN, Zargar SA, et al. Biliary abnormalities associated with extrahepatic portal venous obstruction. Hepatology 17: 807-813, 1993.

6. Nagi B, Kochhar R, Bhasin D, Singh K. Cholangiopathy in extrahepatic portal venous obstruction. Radiological appearances. Acta Radiol 41: 612-615, 2000

7. Malkan GH, Bhatia SJ, Bashir K, et al. Cholangiopathy associated with portal hypertension: diagnostic evaluation and clinical implications. Gastrointest Endosc 49: 344-348, 1999.

8. Bayraktar Y, Balkanci F, Kayhan B, et al. Bile duct varices or "pseudo-cholangiocarcinoma sign" in portal hypertension due to cavernous transformation of the portal vein. Am J Gastroenterol 87: 1801-1806, 1992.

9. Dhiman RK, Behera A, Chawla YK, Dilawari JB, Suri S. Portal hypertensive biliopathy. Gut 56: 1001-1008, 2007.

(C) 2013 The Japanese Society of Internal Medicine http://www.naika.or.jp/imonline/index.html 